AperTO - Archivio Istituzionale Open Access dell'Università di Torino

\title{
Neural bases of the non-conscious perception of emotional signals
}

\section{This is the author's manuscript}

Original Citation:

Availability:

This version is available http://hdl.handle.net/2318/79483

since

Published version:

DOI:10.1038/nrn2889

Terms of use:

Open Access

Anyone can freely access the full text of works made available as "Open Access". Works made available under a Creative Commons license can be used according to the terms and conditions of said license. Use of all other works requires consent of the right holder (author or publisher) if not exempted from copyright protection by the applicable law. 


\section{Neural bases of the non-conscious perception of emotional signals}

Marco Tamietto ${ }^{* \ddagger}$ and Beatrice de Gelder $* \neq \|$

Abstract | Many emotional stimuli are processed without being consciously perceived. Recent evidence indicates that subcortical structures have a substantial role in this processing. These structures are part of a phylogenetically ancient pathway that has specific functional properties and that interacts with cortical processes. There is now increasing evidence that non-consciously perceived emotional stimuli induce distinct neurophysiological changes and influence behaviour towards the consciously perceived world. Understanding the neural bases of the non-conscious perception of emotional signals will clarify the phylogenetic continuity of emotion systems across species and the integration of cortical and subcortical activity in the human brain.

Contemporary neuropsychology and neuroscience are replete with examples of non-conscious perception of visual stimuli, including emotion-inducing ones ${ }^{1}$. Visual stimuli with emotional significance that are not perceived consciously nevertheless induce behavioural and neurophysiological responses that are indicative of a change in emotional state. The notion that emotional events are sometimes not accessible to consciousness has long been familiar, but the last two decades have seen a radical departure from previous psychodynamic conceptualizations associated with Freud and a movement towards evolution-inspired accounts of emotions and consciousness ${ }^{2-7}$ (BOX 1). Non-conscious perception of emotional stimuli must therefore be considered an intrinsic property of the healthy brain and there is now sufficient evidence that such perception is implemented in phylogenetically ancient brain structures ${ }^{5,8}$, mainly in subcortical nuclei. In fact, the evolution of brain structures that are implicated in emotion processing preceded the emergence of neural systems that are involved in sustaining perceptual consciousness. These structures can be studied to tackle the longstanding and unanswered question of what makes a stimulus intrinsically emotional for the brain?.

Here, we first review different examples of nonconscious perception of emotional stimuli. Next, we describe the neural structures composing the subcortical system that underlies these processes in the adult human brain and compare structures and functions of this system across species. Finally, we review evidence that non-conscious perception of emotions can be measured at the behavioural and neurophysiological level, and that it induces changes in cortical and subcortical areas that implement these emotion-related functions, including structures that are involved in action preparation. We argue that non-conscious perception of emotional stimuli is not equivalent to perceiving the same stimuli 'minus' consciousness, but that it is a distinct phenomenon with specific evolutionary benefits.

\section{Types of non-conscious perception}

Only a fraction of sensory input gives rise to conscious perception. Filtering out irrelevant information and keeping only what is relevant for conscious perception is a function traditionally attributed to selective attention ${ }^{10}$. In this Review, we use the term 'attentional unawareness' for the absence of stimulus awareness resulting from attentional selection. However, one may also fail to consciously perceive a stimulus for sensory reasons ${ }^{11}$. For example, if the stimulus energy is too weak (that is, below the detection threshold) or the presentation time too brief (that is, subliminal), the stimulus often does not generate a conscious sensation even when we are paying attention to it $^{12,13}$. In this case we use the term 'sensory unawareness'. Although both phenomena render the observer unaware of the stimulus, they involve different brain processes ${ }^{14}$.

Attentional unawareness. Psychophysical evidence indicates that visual perception of items that lie outside the focus of attention is attenuated or abolished ${ }^{15}$. Accordingly, when attentional resources are engaged in a task, cortical activity that is evoked in visual areas by unattended (that is, task-irrelevant) stimuli is suppressed 
by top-down influences from frontoparietal regions ${ }^{16}$. Emotional stimuli seem to constitute an exception to this effect of attention; indeed, there is extensive evidence from behavioural studies that processing of emotional information is prioritized ${ }^{17}$ (that is, it is less dependent on attentional resources than neutral information). For this reason, emotional stimuli that are task-irrelevant, and ignored, nevertheless interfere with ongoing tasks $^{18,19}$, delay disengagement of attention ${ }^{20}$ and are more easily detected than neutral stimuli - as shown in visual search ${ }^{21,22}$ and attentional blink paradigms ${ }^{23}$ (BOX 2). Notably, however, damage to the amygdala abolishes some of these effects ${ }^{24}$.

Neuroimaging studies in which attention is manipulated using a dual-task design have revealed the brain structures that process unattended emotional stimuli. In most of the studies, stimulus-evoked activity in subcortical structures such as the amygdala is not suppressed when fearful stimuli are unattended ${ }^{25-28}$, but this activity can be reduced when the observer performs a visually demanding task $\mathrm{k}^{29-32}$. Although perception of unattended emotional stimuli is often qualified as non-conscious, it would be more appropriate to define it as pre-attentive (that is, occurring before, and

\section{Box 1 | Consciousness: Facing the terminological jungle}

Consciousness has been one of the most debated issues in the history of psychology and neuroscience. The different notions that attempt to clarify what is meant by non-consciousness tend to reflect different theoretical backgrounds and the methodologies used to investigate awareness. It is useful to make a distinction between 'unconscious' and 'non-conscious'. The first term is rooted in the psychoanalytical tradition and postulates the existence of an active mechanism of psychodynamic suppression of conscious information. By contrast, the use of 'non-conscious' is rooted in the experimental psychology tradition and indicates a perceptual state in which the subject does not report the presence of a stimulus or of one of its attributes (for example, its emotional content) even though there is evidence (behavioural, psychophysiological or neurophysiological) that the stimulus has in fact been processed ${ }^{154}$.

Other terms, such as 'subliminal' or 'implicit' perception, have been used to refer to similar conditions of non-conscious perception. However, 'subliminal' is more frequently encountered in studies of healthy individuals and refers to short or weak stimulus presentations that do not elicit a conscious perception ${ }^{155}$, whereas 'implicit' is a more general term that is sometimes linked with the less restrictive notion of task-irrelevant, involuntary or unintentional processing.

The situation is similar for the term 'automatic', which normally means independence from top-down factors, especially in relation to attention. In the context of attention studies, the term 'pre-attentive' is frequently preferred to 'automatic', as it more clearly indicates that processing of the incoming stimulus occurs before, and independently of, attentional selection. Nevertheless, evidence that a stimulus is processed pre-attentively does not necessarily imply that the observer is unaware of its presence.

The distinction between conscious and non-conscious perception largely depends on the methods used to unravel them. Adopting subjective measures, perception is considered to be non-conscious when participants "claim not be able to discriminate perceptual information at better than chance level"156, irrespective of whether or not their performance (in response to the stimulus) is above chance. Because subjective measures are liable to individuals' response bias, more recent studies have introduced objective measures - some derived from signal detection theory ${ }^{34,58,60,61}$ — that define perception as non-conscious only when the perceptual discrimination is at chance level ${ }^{34,59,60}$. In the present Review we use the terms 'non-conscious perception' or 'perception without awareness' (which we consider to be almost synonymous) to refer to studies that proved, using either subjective or objective measures, that participants cannot report the presence of an emotional visual signal. independently from, attentional selection), unless one can demonstrate that unattended emotional stimuli are also not consciously perceived. This additional check is rarely performed ${ }^{27,28}$, creating uncertainty about whether emotion perception is truly non-conscious in attention-manipulation paradigms.

Research in patients with pathologically limited attention circumvents some of the limitations of studies in healthy individuals. It supports the notion that lack of attention can result in non-conscious perception of emotions and provides additional evidence for the neural basis of non-conscious perception. For example, patients with hemispatial neglect due to right temporoparietal lesions typically fail to pay attention to the contralesional (left) space. Stimuli appearing on the contralesional side often go undetected despite the fact that they are processed normally in bilaterally intact visual areas. Nevertheless, these undetected emotional stimuli can activate emotionsensitive areas in subcortical structures (for example, the amygdala) as well as in portions of the cortex that are directly connected to these structures (for example, the orbitofrontal cortex $)^{33}$, and they can gain access to visual awareness more often than neutral stimuli under identical conditions ${ }^{34-38}$. This effect is reminiscent of the preferential visual processing of task-irrelevant emotions in healthy observers (described above) and presumably engages similar mechanisms.

Sensory unawareness. Backward masking and binocular rivalry (BOX 2) are two key experimental paradigms for investigating sensory unawareness in healthy observers. Both methods interfere with activity in the ventral occipitotemporal cortex, an area that is supposed to be crucial for visual awareness ${ }^{39,40}$. Studies using these paradigms have shown that non-consciously perceived emotional stimuli elicit physiological responses that are indicative of autonomic arousal ${ }^{3,41,42}$, trigger specific electrophysiological components ${ }^{43}$ and induce spontaneous facial muscle activity that reflects the emotion conveyed by the stimulus ${ }^{44,45}$. Neuroimaging studies using backward masking ${ }^{6,746-54}$ or binocular rivalry ${ }^{55-57}$ have shown that non-consciously perceived emotional stimuli elicit activity in the amygdala, superior colliculus, basal ganglia and pulvinar. Activity in subcortical structures is the same, and in several cases even enhanced, in response to non-consciously perceived stimuli compared with activity in response to consciously perceived stimuli ${ }^{25,56}$. Conversely, activity in occipitotemporal, frontal or cingulate areas is typically higher in response to emotional stimuli that are consciously perceived and this activity can be suppressed under conditions of visual unawareness ${ }^{7}$.

It was initially suggested that early studies using subjective measures of participants' awareness possibly overestimated the extent of non-conscious perception of emotional stimuli and identified neural correlates that in fact reflect partial stimulus awareness ${ }^{29,58-60}$. However, more recent studies with very restrictive (that is, objective) criteria for defining visual awareness have supported these early finding $5^{50,52,54,60,61}$. Nevertheless, assessing the neural bases of non-conscious perception 
of emotional stimuli ideally involves a direct comparison between perceived and unperceived, identical stimuli. Unfortunately, evidence of this type is difficult to gather in healthy individuals because the manipulations that

\section{Box 2 | Methods for uncovering non-conscious perception of emotions}

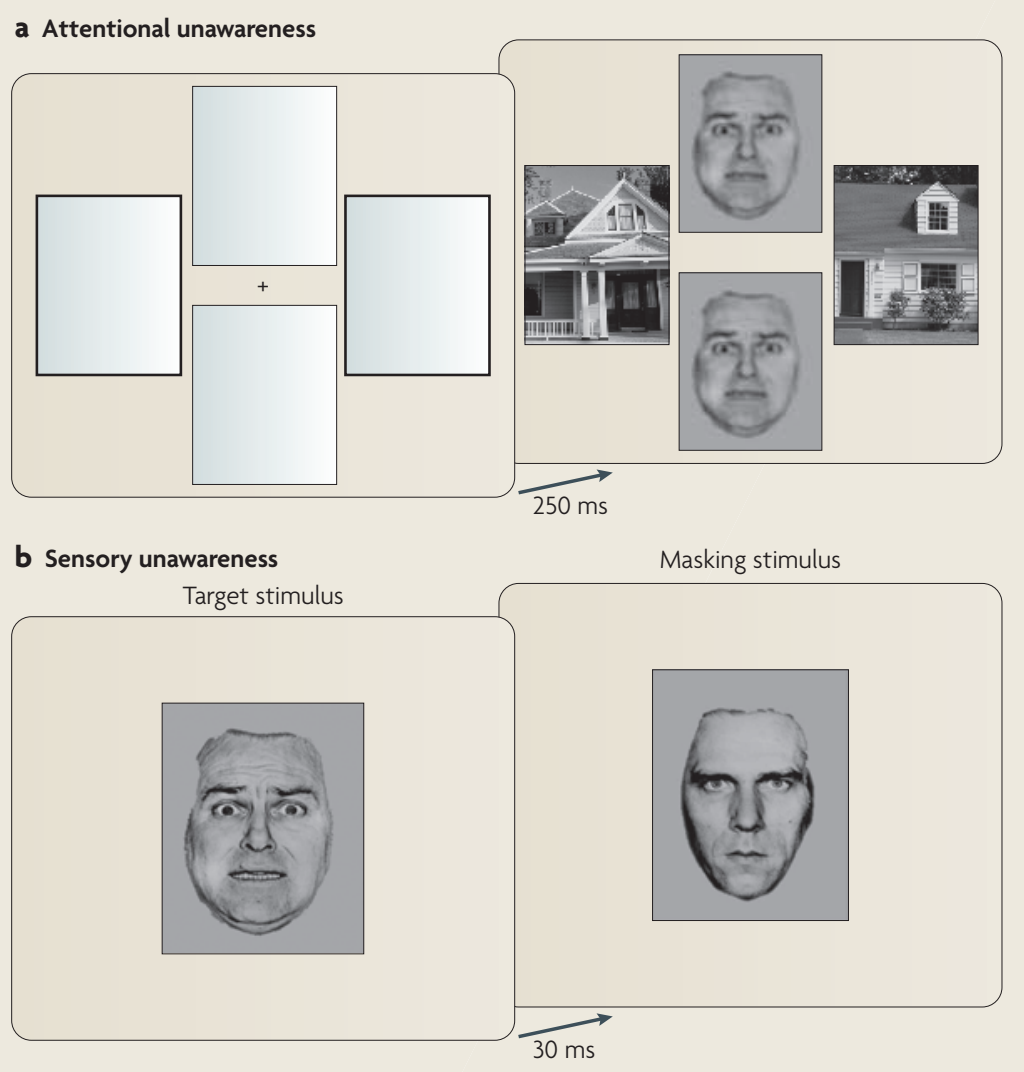

Different methods have been used to induce non-conscious perception of emotions in neurologically intact individuals. Studies of attentional unawareness often use a dualtask paradigm. Here, the subject's attention is absorbed by an attention-demanding task while a secondary emotional stimulus is presented. The unattended emotional stimulus may be presented at the same or at different locations from the primary (and neutral) stimuli. In the first case the effect of object-based attention is investigated, whereas in the second case spatial attention is studied. The face- or house-matching task (see the figure) is a variant of the dual-task paradigm ${ }^{27}$. Here, participants have to judge whether or not the houses are identical while facial expressions appear at irrelevant locations (see the figure, part a). Participants cannot tell whether the ignored faces were fearful or neutral, male or female, or indicate the identity of the faces - an example of attentional unawareness. Another method to induce attentional unawareness is the attentional blink paradigm. Here, a continuous and rapid stream of stimuli is presented at the same location and the subject is instructed to detect a target in the stream. The detection of a first target prevents the detection of a subsequent target for about half a second (the 'blink' period). Sensory unawareness may result from backward masking (see the figure, part b) or from binocular rivalry. In the first case, an initial, emotional target stimulus is briefly presented, immediately followed by a neutral 'masking' stimulus. If the interval between the onset of the target and the masking stimuli is sufficiently brief (typically $<30 \mathrm{~ms}$ ) the subject is unaware of the first stimulus and only reports the second (neutral) stimulus ${ }^{3,7}$. In binocular rivalry paradigms different images are presented to the corresponding regions of the two eyes. Under these conditions monocular channels in the primary visual cortex (V1) alternatively inhibit each other so that one of the two images dominates perception at any given moment and is consciously perceived, whereas the other image is suppressed and does not reach awareness ${ }^{52}$. Spontaneous alternations of which stimulus is consciously perceived occur, with each dominant percept lasting a few seconds. Part a is modified, with permission, from REF. 27 (c) (2001) Cell Press. are used to render a stimulus 'invisible' inevitably also render the stimulus spatially and temporally different from its consciously visible counterpart.

At present, studies of patients with cortical blindness following destruction of the visual cortex provide the best opportunity to clarify the neural basis and properties of non-conscious perception of emotional stimuli. These patients are permanently blind to stimuli presented inside the scotoma (the visual field region affected by the cortical lesion), including suprathreshold and long-lasting stimuli ${ }^{62}$. Therefore, cortical blindness creates a sharp distinction between conscious and non-conscious perception due to sensory, as opposed to attentional, causes. These patients are able to discriminate emotional stimuli that they report not to have seen, for example by 'guessing' whether the stimulus expresses happiness or fear ${ }^{2}-$ a phenomenon known as affective blindsight ${ }^{63}$. Their behavioural fluency in this task is associated with activity in several subcortical structures that were also active in studies in which nonconscious perception of emotional stimuli resulted from experimental manipulation ${ }^{2,64-69}$.

A caveat is needed in the case of patients because post-lesion and experience-dependent plasticity may have enhanced the sensitivity of spared subcortical nuclei to visual and emotion attributes. Applying transcranial magnetic stimulation (TMS) over the visual cortex of healthy participants can create 'virtual' cortical lesions, leading to transient blindness without neuronal reorganization ${ }^{70}$. The only such TMS study to date showed that blocking the cortical visual route interrupts conscious perception but does not suppress non-conscious perception of emotional stimuli ${ }^{71}$, as participants were able to discriminate between sad and happy expressions and locate their position in a fouritem array. This suggests that subcortical contributions to non-conscious perception of emotional stimuli are not dependent on post-lesion plasticity and also occur in the intact brain.

Comparing attentional and sensory unawareness. Studies on attentional and sensory unawareness in healthy observers have provided evidence that conscious and non-conscious modes of perceiving emotional stimuli coexist. Beyond these observations, patient studies offer the opportunity to reveal which brain structures are necessary for - rather than simply involved in conscious and non-conscious perception of emotional stimuli. Combined evidence from brain imaging and patient studies indicates that sensory and attentional unawareness are qualitatively different ${ }^{12,14}$. In sensory unawareness the stimulus strength is insufficient to evoke cortical activity in visual areas. Nonetheless, the same stimuli can trigger activity in subcortical visual structures, such as the superior colliculus, that have a lower threshold of activation ${ }^{13,72}$. This is the appropriate condition under which to study the structures that compose the core system for the non-conscious perception of emotional stimuli because the stimulus has not already been processed in the striate cortex (also known as the primary visual cortex; V1). In the case of attentional 
unawareness, the emotional stimulus has entered the cortical visual system, starting with V1, and is potentially accessible to consciousness but does not actually gain access to awareness because attention is allocated elsewhere, thereby reducing cortical responsivity to the unattended stimulus ${ }^{16}$. This case is the best suited for understanding at what stage along the informationprocessing stream the emotional content is extracted from a stimulus and how structures that are involved in non-conscious perception of emotional stimuli modulate cortical activity in visual and attentional areas.

A limitation in our present understanding of attentional and sensory unawareness for emotional stimuli concerns the prevailing use of stimuli that are related to danger (that is, fearful and angry expressions), and it is still disputed whether signals conveying other emotions engage similar processes and structures. However, recent data indicate partly similar, though weaker, effects when non-conscious perception of happiness, sadness or disgust is tested ${ }^{38,48,49,64,68}$.

\section{Functional and structural neuroanatomy}

Human adult brain: topological aspects. Different subcortical structures are involved in non-conscious perception of emotional stimuli (FIG. 1). Several of these

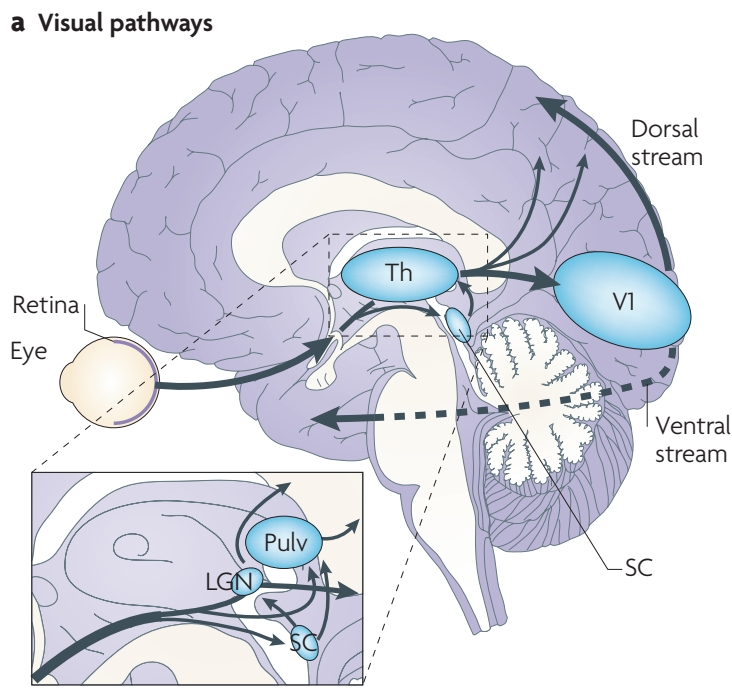

structures have been investigated extensively and their functions are reasonably well understood, whereas the involvement of other areas became apparent only recently and requires further study. At present, a distinction can be made between two subsystems. The first consists of structures that are involved in the visual encoding of emotional stimuli and that are directly connected to subcortical centres that include visually responsive neurons. This system entails a pathway including the superior colliculus, the visual pulvinar, the amygdala, the substantia innominata and the nucleus accumbens. The second is a network that encompasses subcortical areas that are recruited for non-visual emotion-related functions, such as emotional reactions, memory consolidation, motivation and disposition tendencies. This second network mainly includes the locus coeruleus, the periaqueductal grey, the nucleus basalis of Meynert, parts of the basal ganglia, the hypothalamus and the hippocampus.

The superior colliculus is a laminar brainstem structure that receives direct projections from retinal ganglion cells with large receptive fields and with rapidly conducting axons that form the magnocellular pathway ${ }^{73}$. Superior colliculus activity is largely unaffected by lesions of cortical visual areas ${ }^{74}$ and responds to non-consciously perceived emotional stimuli in normal individuals $s^{50,51,53}$

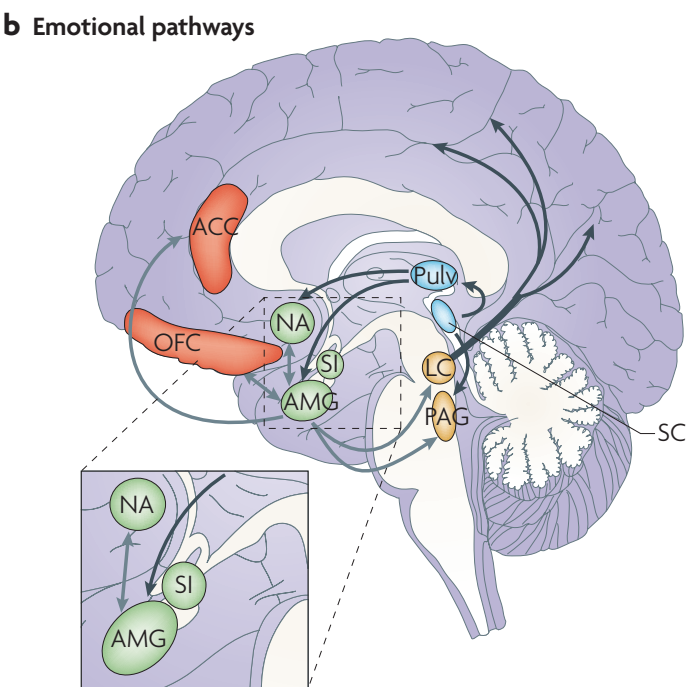

Figure 1 | Cortical and subcortical pathways for vision and emotion. a | The primary visual pathway (shown by thick arrows) originates from the retina and projects to the primary visual cortex (V1) in the occipital lobe via an intermediate station in the lateral geniculate nucleus (LGN) of the thalamus (Th). From V1, visual information reaches the extrastriate cortex along the ventral (occipitotemporal) and the dorsal (occipitoparietal) stream. However, a minority of fibres originating from the retina take a secondary route (shown by thin arrows) and reach both the superior colliculus (SC) and the pulvinar (Pulv). These two subcortical sites are connected and also send direct projections to the extrastriate visual cortex, bypassing V1. Another V1-independent visual pathway consists of the direct projections between the superior colliculus and the LGN that, in turn, send efferents to extrastriate cortices in the dorsal stream. $\mathbf{b}$ | The 'emotion system' includes several cortical and subcortical areas. Among the subcortical structures are the amygdala (AMG) and the substantia innominata (SI; shown in green), which are buried deeply in the temporal lobe and in the basal forebrain, respectively, the nucleus accumbens (NA) in the basal ganglia (shown in green) and brainstem nuclei (shown in yellow), such as the periaqueductal grey (PAG) and the locus coeruleus (LC). Among cortical areas (shown in red) are the orbitofrontal (OFC) and the anterior cingulate cortex (ACC). The visual and emotional systems are extensively interconnected, especially at the subcortical level, where the superior colliculus is connected to the amygdala via the pulvinar. Direct connections also exist between subcortical and cortical emotion regions (for example, between the amygdala and OFC or ACC), between subcortical structures for emotions and cortical visual areas (for example, between the amygdala and temporal cortex) (not shown) and between brainstem nuclei and the cortex via diffuse projections (shown only from the LC). Grey arrows indicate connections within the emotion system. neurons that originates from retinal ganglion cells with large receptive fields and that is characterized by low spatial resolution and rapid

transmission of nerve impulses. 
and in patients with cortical blindness ${ }^{64,66,75}$. The superior colliculus is the earliest post-retinal subcortical structure that responds to coarse (that is, low spatial frequency) emotional stimuli.

The pulvinar is responsive to salient visual targets and is active during non-conscious perception of emotional stimuli ${ }^{64,66,75}$. It comprises several retinotopicallyorganized subnuclei and its lateral and inferior parts receive direct projections from the retina and from the superior colliculus ${ }^{76}$. The pulvinar is also involved in attentional mechanisms and its lesion abolishes the automatic 'attention-grabbing' effects of consciously perceived emotional stimuli ${ }^{77}$. It is unclear, however, whether this effect of pulvinar damage indicates a direct role of the pulvinar in the perception of emotional stimuli or an interference with the relay of visual information to the amygdala, with which the pulvinar is monosynaptically connected ${ }^{78}$.

The amygdala is perhaps the most extensively studied subcortical structure that is involved in emotion processing, particularly with respect to its role in the perception and response to danger ${ }^{79,80}$. Contrary to the traditional view, positively valenced emotional stimuli, like happy or surprised expressions, can also activate the amygdala ${ }^{49,81,82}$ but with a qualitatively different response profile that entails more activity in ventral parts and only at later stimulus presentations compared to fearful expressions ${ }^{82}$. Amygdala activation in response to emotional stimuli has been reported under conditions of sensory ${ }^{6,7,46,48-56}$ and attentional unawareness ${ }^{25-28}$ in neurologically intact observers and in brain-damaged patients with cortical blindness or hemispatial neglect. The amygdala is involved in both conscious and nonconscious perception of emotional stimuli (unlike the superior colliculus and pulvinar ${ }^{81}$. This dual role is probably related to the fact that the amygdala is a complex system (it includes up to 12 subnuclei) and receives visual information from different pathways - one originating in the sensory cortex and one originating in subcortical areas ${ }^{79}$. Therefore, a better understanding of the amygdala's functions in non-conscious perception of emotional stimuli can be achieved by considering the different sensory networks of which the amygdala is part and by focusing on its connectivity, as discussed below.

The substantia innominata is a sublenticular portion of the basal forebrain and comprises several intermingled neuronal groups — such as the sublenticular extended amygdala and the bed nucleus of the stria terminalis that represent an extension of the dorsal amygdala ${ }^{83,84}$. Activity in the substantia innominata may be triggered by backwardly masked emotional stimuli ${ }^{7}$, but its response is probably secondary to amygdala activity. Moreover, there is currently no evidence about the involvement of the substantia innominata during non-conscious perception of emotional stimuli in patients with cortical blindness or about its role in attentional unawareness. Several studies have reported activity in the substantia innominata in response to consciously perceived emotional stimuli ${ }^{84,85}$. This activity differs from the amygdala, however, in that it seems to respond more to the arousal rather than the emotional valence of the stimuli ${ }^{85,86}$.
The nucleus accumbens (or ventral striatum) has been implicated in reward processing and it is activated even by non-consciously perceived omissions of expected rewards ${ }^{87}$. This is consistent with initial findings that suggested a connection from the superior colliculus to the striatum via the pulvinar and with the presence of a dopaminergic output pathway to the substantia nigra ${ }^{88,89}$.

The periaqueductal grey and the locus coeruleus are implicated in relatively automatic and reflex-like defensive responses ${ }^{90}$. The periaqueductal grey is located close to the superior colliculus, from which it receives visual information. The locus coeruleus is a source of noradrenergic activation and it modulates cortical activity ${ }^{91}$ (for example, in the anterior cingulate and ventral prefrontal regions), as well as activity in subcortical structures, such as the amygdala, pulvinar and superior colliculus, in response to non-consciously perceived emotions ${ }^{50}$. A similar function has been postulated for other nuclei in the substantia innominata, such as the nucleus basalis of Meynert, which receives afferents from the amygdala and projects to widespread cortical areas under cholinergic modulation ${ }^{92}$. The basal ganglia are involved in automatic motor responses and in conscious emotion recognition ${ }^{93,94}$. One study also reported basal ganglia activity for backwardly masked expressions of disgust ${ }^{95}$. The hypothalamus has been implicated in consumptive behaviours and in homeostasis ${ }^{96}$ and is part of an extensive reward network that also includes the amygdala and ventral striatum. Finally, the hippocampus is involved in the contextual evaluation of emotional stimuli and works together with the amygdala in mediating implicit learning and memory consolidation for consciously and non-consciously perceived emotional stimuli ${ }^{6}$.

Human adult brain: network aspects. Recent neuroimaging data have clarified the functional connectivity between several subcortical structures that are involved in non-conscious perception of emotional stimuli. The superior colliculus, pulvinar and amygdala constitute a functional network that shows increased, positive covariation of activity in response to non-consciously perceived fearful facial expressions ${ }^{50,51,53}$. By contrast, the major cortical pathway relaying visual input to the amygdala - starting from the V1 and terminating in the inferotemporal cortex that provides the direct connection to the amygdala ${ }^{79,80}$ - does not show substantial activity and functional connectivity under the same conditions but does so during conscious perception of emotional stimuli ${ }^{53,55}$. These results suggest that a functional subcortical pathway to the amygdala is engaged during non-conscious fear perception. Similar findings have been reported in patients with cortical blindness in response to non-consciously perceived facial and bodily expressions of fear and happiness and support the notion of a functional segregation between subcortical and cortical pathways ${ }^{64,66,75}$.

Covariations in functional responses do not necessarily indicate direct anatomical connectivity among the implicated regions. The anatomical connections between the structures that form the subcortical system for 
non-conscious perception of emotional stimuli have been studied in primate and non-primate animals (see below), but almost nothing is known about these connections in the human brain. A recent diffusion tensor imaging (DTI) study, involving a patient with cortical blindness and five age-matched healthy observers ${ }^{97}$, showed direct connections between the structures of the subcortical network (that is, between the superior colliculus and amygdala via the pulvinar) in all subjects. Notably, however, the number of fibres in the patient with cortical blindness was increased and the shape of the fibre bundle was different, forming a loop between the three structures that was absent in normal subjects ${ }^{97}$.

On the basis of studies in animals, two functional properties have been proposed and tested for this subcortical system: first, it provides a rapid analysis of the emotional attributes of the stimuli; and, second, it is responsive to the more coarse aspects of the stimulus (for example, its global shape and configuration) ${ }^{5}$. Electroencephalography (EEG) and magnetoencephalography (MEG) studies have provided mixed results regarding the speed of processing in the subcortical system, with some studies showing fast processing of emotional stimuli before any fine-grained analysis or attentional modulation can occur ${ }^{98,99}$, and others showing longer latencies ${ }^{100}$. A recent study that combined MEG and MRI methods reported early, event-related synchronization in the posterior thalamus (probably in the pulvinar), as fast as $10-20 \mathrm{~ms}$ after onset of the presentation of fearful facial expressions, followed by eventrelated synchronization in the amygdala at $20-30 \mathrm{~ms}$ after onset ${ }^{101}$. By comparison, synchronization in the striate cortex occurred only $40-50 \mathrm{~ms}$ after stimulus onset. With respect to the second functional aspect, responses in the superior colliculus and pulvinar are tuned to coarse information in low spatial frequencies because these structures receive visual information from the magnocellular pathway ${ }^{73}$. By contrast, cortical areas in the ventral visual stream receive visual information predominantly from the parvocellular pathway, which provides high spatial frequency signals ${ }^{102}$. Consistent with these neurophysiological findings, the subcortical pathway to the amygdala is specifically sensitive to the presentation of fearful faces in low spatial frequencies ${ }^{103}$.

Homologous pathways in animal brains. Studies in rats and birds have demonstrated that a subcortical pathway for fast processing of coarse emotional stimuli exists in the auditory and visual domains (FIG. 2). This pathway encompasses the colliculus, pulvinar and amygdala. For example, in birds the optic tectum (an analogue to the mammalian superior colliculus) and the nucleus rotundus (an analogue to the visual pulvinar) are directly connected and relay visual information to the amygdala (also known as the taenia) ${ }^{104}$. After lesions to the optic tectum chicks cease to show a startled or excited response to behaviourally significant visual stimuli, and can delay avoidance and attack responses, indicating the functional relevance of this pathway for emotion processing ${ }^{105}$. Likewise, in rats there are direct connections from the superior colliculus to thalamic neurons that project to the lateral nucleus of the amygdala, forming a subcortical pathway for the transfer of visual information to the amygdala ${ }^{106}$. Lesions to the rat superior colliculus slow down behavioural reactions to threatening stimuli, suggesting that the cortical pathway to the amygdala can carry out functions that are normally performed by the direct subcortical route, but at the expense of reaction speed ${ }^{107}$.

In non-human primates, the role of the amygdala in emotion perception (and other social processes) has been repeatedly documented since Weiskrantz first showed that bilateral lesions to the amygdala were sufficient to induce a loss of reactivity to emotional visual stimuli ${ }^{108}$. However, it is disputed whether there are direct connections between the areas that form the subcortical pathway for processing emotional stimuli in the monkey brain (see REF. 29 for a review). Although the superior colliculus projects to the inferior parts of the pulvinar ${ }^{76}$, and the amygdala receives projections from the medial pulvinar ${ }^{109}$, direct connections between the inferior and medial nuclei of the pulvinar remain to be described. Moreover, the superior colliculus also projects directly to the intercalated layers of the lateral geniculate nucleus ${ }^{110}$, and it was shown recently that
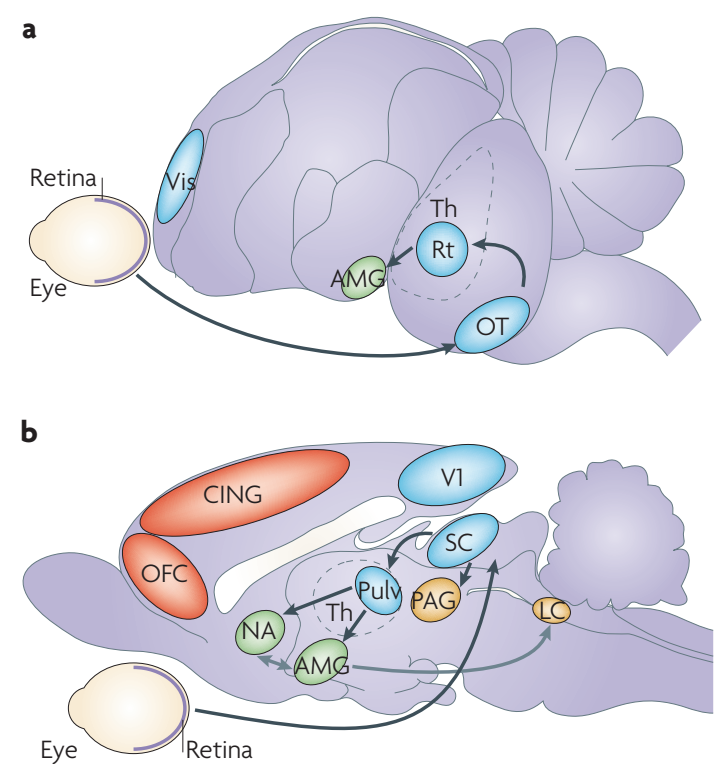

Figure 2 | Subcortical pathway for processing emotional signals in the animal brain. a | Lateral view of the avian brain showing direct connections between the retina and the optic tectum (OT) (an analogue of the mammalian superior colliculus (SC)), the optic tectum and the nucleus rotundus ( $\mathrm{Rt}$; an analogue of the visual pulvinar (Pulv)) in the thalamus (Th), and the amygdala (AMG). The visual Wulst area (Vis; the analogue to the mammalian primary visual cortex) is also displayed. b | Sagittal view of the rat brain showing the equivalent pathway from the retina to the superior colliculus and from there to the pulvinar and the amygdala. Other subcortical and cortical structures that are involved in emotion processing are also displayed. These include the cingulate cortex (CING) and the orbitofrontal cortex (OFC), the nucleus accumbens (NA), and, in the brainstem, the periaqueductal grey (PAG) and the locus coeruleus (LC). V1, primary visual cortex. 


\section{Box 3 | Ontogenetic development of the subcortical pathway for the perception of emotional signals}

The visual system in newborns seems to be more sensitive to low spatial frequency stimuli than in adults. This is reflected in the well-documented preference for face detection in newborn babies, as face configurations are easily recognizable in the low-frequency range ${ }^{157}$. At around the age of 5-7 months, infants start to exhibit stable visual discrimination of different facial expressions ${ }^{158}$ and to show the typical effects that emotional stimuli exert on visual and attentional mechanisms in adults. These effects encompass the preference to look at fearful over neutral or happy faces, enhanced visual and attention-related ERP components ${ }^{99}$, and difficulties in disengaging attention from faces showing fearful expressions $^{159}$. Facial expression preferences seem to be mediated by the subcortical pathway, which includes the superior colliculus, pulvinar and amygdala ${ }^{115}$. This is consistent with findings from monkeys showing that the formation of these structures and their connections with the cortex are more complete at birth compared with the relatively immature development of the cortical visual system ${ }^{160}$, which also shows higher levels of plasticity and neural reorganization during development ${ }^{161}$.

Subcortical orientation to, and processing of, stimuli such as faces in the early stages of development may represent the starting point for the later specialization of portions of the visual cortex to specific categories of emotionally relevant stimuli such as faces (in the fusiform face area) ${ }^{147}$ or bodies (in the extrastriate body area) ${ }^{148}$. From this perspective ${ }^{115}$, the early functioning of the subcortical system would ensure that infants preferentially orient to categories of visual stimuli that potentially convey emotion information. This induces foveation to such stimuli more often than to others, thereby providing a more frequent input to the visual cortex. In addition, the modulatory influence of subcortical structures over the visual cortex can result in enhanced cortical activity in response to faces or bodies and this further accelerates cortical specialization. Consistent with the proposed role of the subcortical system during development, atypically developing children with autism, who have amygdala dysfunctions ${ }^{162}$, seem to depend more on high spatial frequency aspects for processing faces than typical individuals ${ }^{163}$. As is the case with other development mechanisms in the brain, it is likely that newborns' sensitivity to emotional expressions results from the combination of an experience-independent system - located in subcortical structures - that is biased to process coarse emotional stimuli and experiencedependent factors that promote functional coupling of these structures with visual and attentional areas in the cortex, by refining and stabilizing initially existing cortico-subcortical synaptic connections ${ }^{113}$.

inactivation of the lateral geniculate nucleus abolishes residual visual abilities in monkeys with V1 lesions ${ }^{111}$. This raises the possibility, which has not been explored directly thus far, that visual information might also reach the amygdala through the lateral geniculate nucleus analogous to the direct connections between the medial geniculate nucleus and the lateral amygdala that have been reported in the auditory domain ${ }^{5,79}$.

Data from studies in humans and animals are beginning to reveal the continuity of subcortical emotion processing across species and its evolutionary role in shaping adaptive behaviour. A recent proposal suggests that fear detection has played a major part in shaping the visual system of primates and in its integration with an emotion circuit that is centred on the amygdala. According to this proposal ${ }^{112}$, snakes presumably represented a major threat for our ancestors and the need to detect them on the ground accelerated the development of greater orbital convergence, allowing better shortrange stereopsis, particularly in the lower visual field. The koniocellular and magnocellular pathways from the retina further developed to connect the superior colliculus with the pulvinar, thereby promoting fast and automatic detection of snakes. This mechanism presumably became generalized for detection of other fear-relevant stimuli. The same proposal suggests that the fast reactivity of this subcortical pathway to visual stimuli with low resolution has also promoted the parallel development of a cortical visual pathway to the amygdala with complementary features to those of the subcortical pathway ${ }^{112}$. The cortical pathway seems to have drawn on parvocellular input that enabled trichromacy and fine-grained foveal vision, which may have served to facilitate detailed, slow and conscious analysis of visual stimuli to aid deliberate actions.
Consistent with evidence that the subcortical pathway for processing emotional stimuli emerged early in phylogenesis, recent evidence in human and non-human primates also indicates that the formation of these same structures is more developed at birth compared to the relatively immature development of the cortical areas involved in visual and emotional processing ${ }^{113}$ (BOX 3).

\section{The role of non-conscious perception}

Non-conscious perception of emotional stimuli has behavioural consequences and is accompanied by characteristic neurophysiological correlates of changes in the emotional state of the (unaware) observer. These behavioural and neurophysiological outcomes are often qualitatively and quantitatively different from those associated with conscious perception, suggesting that non-conscious perception of emotional stimuli is not simply a degraded counterpart of conscious perception but a different mode of processing visual signals.

A system specialized for emotional signals, not only for faces. Which stimulus properties trigger non-conscious perception of emotional stimuli? Research on emotion perception with and without awareness has concentrated on facial expressions ${ }^{114}$. This has contributed to the prevailing assumption that the subcortical pathway is specialized for detecting emotional expressions on faces rather than for detecting emotional stimuli per $s^{115}$. However, the inability of patients with cortical blindness to correctly guess non-emotional facial attributes, such as personal identity, speaks against this assumption ${ }^{116}$. Likewise, facial expressions of complex social emotions, like arrogance or guilt, seem to require processing in higher visual areas that are associated with visual awareness ${ }^{117}$. 
Recent findings suggest that not just a person's face but their whole body posture can trigger non-conscious perception of the person's emotions in an observer ${ }^{118,119}$. It therefore makes sense to assume that, like facial expressions, body postures belong to a category of biological stimuli to which we are evolutionarily prepared to respond. Non-conscious perception of body expressions indicating fear or joy activates the pulvinar and can induce arousal or mimicry responses in healthy individuals and in patients with cortical blindness ${ }^{45,64,68}$, whereas unattended angry or fearful bodily expressions can activate the amygdala and summon attention in healthy individuals ${ }^{119}$ and patients with hemispatial neglect ${ }^{34}$.

Stimuli that represent evolution-determined threats, such as spiders and snakes, have also been tested under conditions of visual unawareness. These stimuli induced enhanced physiological arousal and amygdala activity $^{120-122}$, particularly in individuals who were phobic to these types of stimuli, and summoned attention in patients with hemispatial neglect ${ }^{37}$. By contrast, showing complex affective scenes ${ }^{123}$ (for example, cockroaches or houses on fire) to cortically blind patients did not have such effects; the patients were unable to guess the emotional content of these scenes. This suggests that the analysis of the emotional content of complex scenes may depend on conscious visual perception and cortical processing, although further research is needed to confirm this.

Autonomic and expressive changes. Non-conscious perception of emotional stimuli is generally associated with more rapid and intense responses in terms of physiological changes and facial expressions (in the observer) than conscious perception of emotional stimuli. This suggests an inverse relationship between stimulus awareness and its impact on behavioural and neurophysiological reactions.

Physiological changes that are associated with nonconscious perception of emotional stimuli include enhanced skin conductance ${ }^{3,41}$, increased frequency of eye blink (indicating startle reactions or avoidance) ${ }^{65}$, changes in stress hormone levels ${ }^{124}$, increased pupil dilation $^{68}$ and heart rate changes ${ }^{125}$. These changes index arousal and their function is to prepare the organism for reacting to impeding and salient events.

In the unaware observer, undetected emotional stimuli can also induce spontaneous facial reactions that reflect the affective valence of the stimuli, as recorded using electromyography $(\mathrm{EMG})^{45,68}$. This spontaneous tendency to synchronize our facial expressions with the emotional meaning of other individuals' expressions is likely to play a part in social interactions ${ }^{126}$. Spontaneous facial expressions are served by the extrapyramidal motor system in which the basal ganglia have a major role, whereas intentional expressions are mediated by the pyramidal system, which is under cortical control ${ }^{127}$. A recent study has measured psychophysiological and expressive changes associated with conscious and non-conscious perception of facial and bodily expressions of fear and happiness in patients with cortical blindness ${ }^{68}$. Results showed that facial reactions were faster and autonomic arousal was higher for non-consciously than for consciously perceived stimuli (FIG. 3). This is in line with data showing that physiological and expressive responses can be stronger ${ }^{128}$ and faster ${ }^{42}$ when triggered by emotional signals that remain inaccessible to awareness.

\section{Neural integration and awareness}

The findings described above indicate that nonconscious perception of emotional stimuli is associated with the functional integrity and activity of several subcortical structures, which seem to function independently of cortical areas. This raises the question of whether the distinction between subcortical and cortical processes simply overlaps with the distinction between non-conscious and conscious perception. Two alternative proposals have been put forth ${ }^{129}$. The first proposal is that conscious and non-conscious perception have the same neural correlates but that in the case of vision without awareness the evoked brain activity is simply less than for consciously perceived stimuli. The difference is thus a matter of quantity of neural activity. This view is supported by evidence that higher-order visual areas, such as the parahippocampal gyrus, the fusiform gyrus or the middle temporal area (MT; also known as visual area V5), are activated by category-specific stimulus properties (for example, the presence of a house, a face or a moving stimulus, respectively) regardless of whether or not the stimuli are consciously perceived, but with higher levels of cortical activation in the case of conscious perception ${ }^{130-132}$. A second proposal is that there are separate pathways for conscious and nonconscious vision that already diverge from relatively early processing stages. Here, the difference between neural systems sustaining vision with and without awareness is qualitative. This second proposal is indirectly supported by psychophysical evidence showing that conscious and non-conscious perception have different sensory thresholds ${ }^{13,133,134}$ and, more directly, by neuroimaging evidence that visual awareness is associated with activity in temporal or prefrontal cortices ${ }^{135,136}$, whereas nonconscious perception induces activity in subcortical or early sensory areas ${ }^{137-139}$. Notably, however, the two theories overlap in assuming that visual consciousness is linked to enhanced or exclusive activity (according to the former and latter hypotheses, respectively) in several crucial cortical areas; either in the occipitotemporal visual cortex alone or in association with frontoparietal networks that exert top-down amplification over sensory areas ${ }^{12}$.

It is worth considering a third alternative that, although primarily derived from emotion studies, seems to fit better with the data concerning visual awareness of neutral stimuli. We propose that the neural pathways for conscious and non-conscious perception of emotions are not entirely different and are not completely segregated. A major difference between the two types of perception may be the combined involvement of cortical areas and of cortico-subcortical interactions when stimuli are consciously perceived. The dichotomy of conscious and non-conscious perception of emotional signals can thus be reformulated in neural terms as the integration 
of activity in subcortical and cortical structures. Fastlatency responses supported by excitatory feedforward connections along the colliculus-pulvinar-amygdala pathway may be too rapid and/or too weak to engage the cortex and to generate the re-entrant cortico-subcortical feedback that is associated with visual awareness ${ }^{53}$. Indeed, these back-projecting neurons from cortical to subcortical areas probably have higher activation thresholds than the forward-projecting neurons in subcortical sites and therefore may not be activated by the weak neural response evoked by non-consciously perceived stimuli $^{13,133,134}$. As this cortical feedback might reflect inhibitory modulation over subcortical areas ${ }^{140}$, its absence during non-conscious perception of emotional stimuli could also explain the apparently paradoxical finding that subcortical activity can be enhanced, rather than being unchanged or reduced, during nonconscious compared to conscious perception of emotional stimuli ${ }^{25,56}$. Therefore, our hypothesis is also consistent with dynamic models that consider re-entrant cortical feedback - at slow timescales and synchronized in the gamma-band frequency - to be critical to the emergence of visual awareness ${ }^{141}$. This dynamic integration may result from the excitatory action of subcortical over cortical areas and the inhibitory activity cortical over subcortical structures.

This third alternative seems to be supported by several behavioural and neurophysiological findings reported here. At the behavioural level, unattended or non-consciously perceived emotional stimuli can interfere with an explicit ongoing task - for example, by disrupting performance ${ }^{18,19}$, by delaying or speeding up responses to a simultaneously present and consciously perceived emotional signal (depending on the emotional congruence between the two stimuli $\left.{ }^{60,69}\right)$, by affecting the disengagement of attention ${ }^{20}$ or by altering perceptual sensitivity ${ }^{34}$. Likewise, attitudes and preferences towards neutral stimuli may be shifted towards
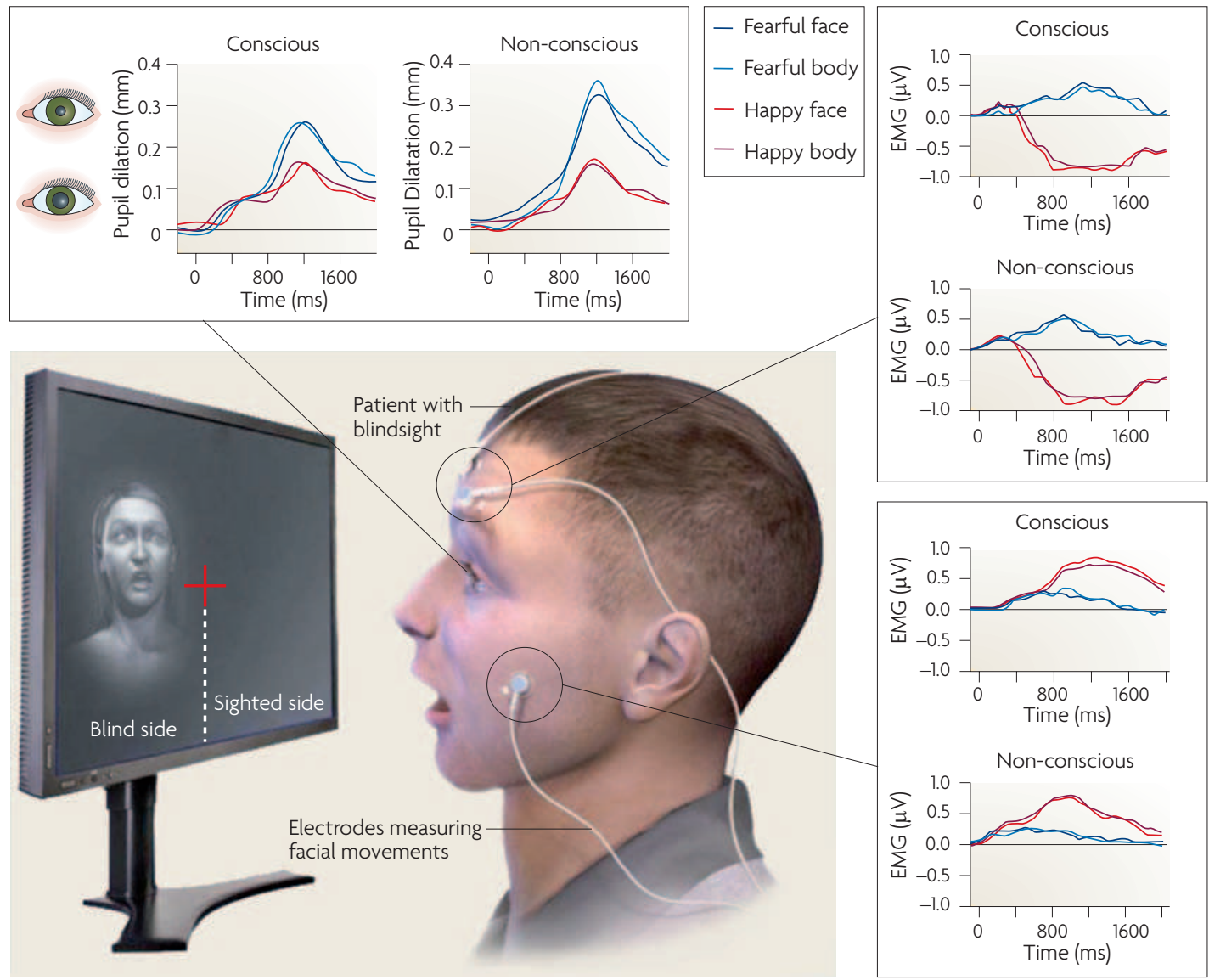

Figure 3 | Expressive and autonomic changes induced by non-conscious perception of emotional stimuli.

Spontaneous facial and pupillary reactions can be triggered by passive exposure to consciously and non-consciously perceived expressions of fear and happiness in patients with cortical blindness and 'affective blindsight'. The zygomaticus major muscle (which is involved in smiling) is activated to a greater extent by conscious and non-conscious perception of happy expressions than by conscious and non-conscious perception of fearful expressions, whereas the corrugator supercilii (which is involved in frowning) is activated by conscious and non-conscious perception of fearful expressions more strongly than by perception of happy expressions. Notably, facial reactions are faster and pupil dilation (indicative of autonomic arousal) is higher for non-consciously than for consciously perceived facial and bodily expressions. Data from REF. 68. Image reproduced, with permission, from REF. 63 @ (2010) Macmillan Publishers Ltd. All rights reserved. EMG, electromyography. 


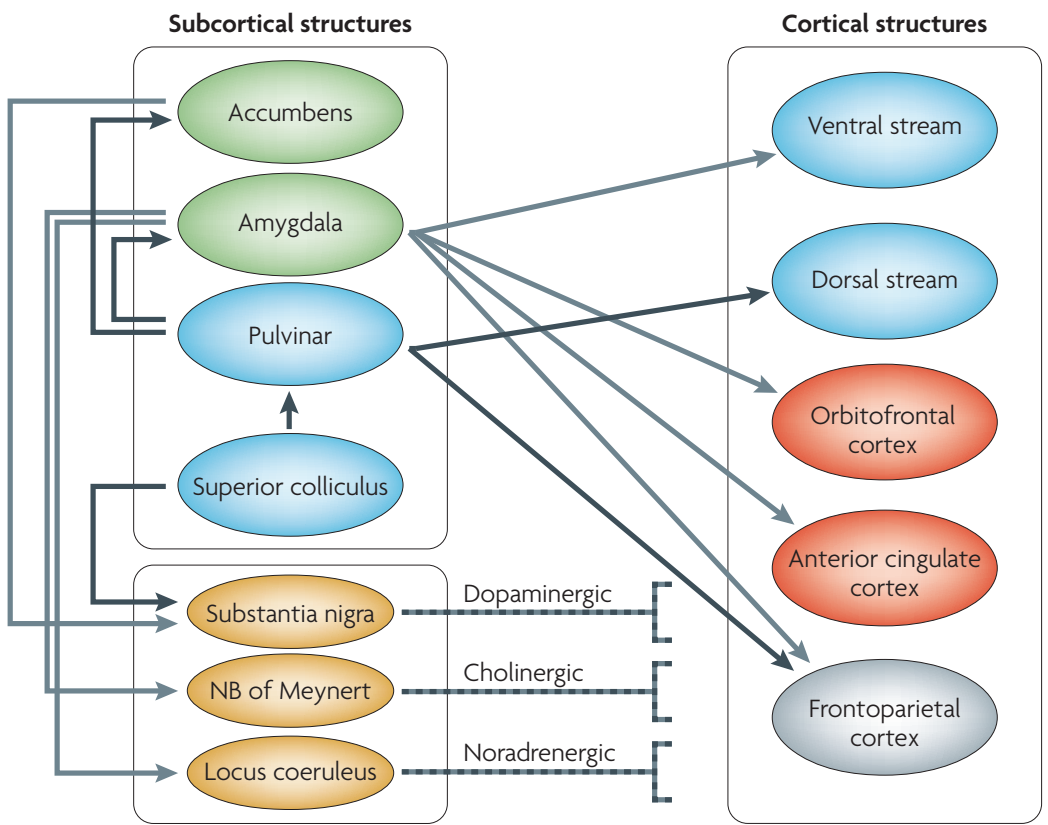

Figure 4 | Circuits for subcortical modulation of cortical activity. Subcortical structures that are involved in non-conscious perception of emotions can modulate cortical activity either directly or indirectly. The amygdala has direct connections to cortical visual areas in the ventral stream, to the orbitofrontal and anterior cingulate cortices, which are involved in conscious perception of emotions, and to the frontoparietal network, which is involved in attention. The pulvinar has direct connections to the visual cortex in the dorsal stream, to the frontoparietal network and to the amygdala. Subcortical structures can also modulate cortical activity indirectly through downstream connections to other sites in the basal forebrain and in the brainstem that project to widespread cortical regions. To simplify the diagram only the most relevant connections discussed in the text are shown. Emotion-related subcortical areas are shown in green; areas involved in visual processing are shown in blue; emotion-related cortical areas are shown in red; brainstem areas are shown in yellow. NB of Meynert, nucleus basalis of Meynert. destruction of the superior colliculus ${ }^{146}$. Therefore, these direct mechanisms also affect cortical visual processes when the emotional stimulus is perceived nonconsciously, modulating extrastriate functions in a precise, category-specific, fashion ${ }^{147,148}$. In line with this, enhanced processing of emotional stimuli in the extrastriate cortex is abolished by lesions to the amygdala that leave the visual cortex intact ${ }^{149}$. Moreover, the amygdala has direct connections to cortical areas related to conscious perception of emotional stimuli, such as the orbitofrontal and cingulate cortices ${ }^{80}$, thereby providing a likely site of convergence between conscious and non-conscious perception. Finally, subcortical structures can also modulate cortical activity and influence conscious perception indirectly through downstream connections to different sites in the basal forebrain and brainstem that project to widespread cortical regions, including visual areas and frontoparietal regions that are involved in attentional control. These indirect routes encompass the dopaminergic pathway from the substantia nigra, the noradrenergic pathway involving the locus coeruleus and the cholinergic system, to which the nucleus basalis of Meynert belongs.

\section{Outstanding questions}

Not surprisingly, the major outstanding questions that are raised by non-conscious perception represent challenges to current cortical theories of consciousness, of emotion and subjective experience.

Do observers who show non-conscious perception of emotional signals actually experience their own emotional changes consciously? Studies into neurophysiological and bodily changes associated with emotions are classical sources of evidence for understanding the relationship between an emotional state (that is, the objective functional state of the person) and the conscious experience of being in such a state (that is, the feeling associated with the emotional state $)^{150}$. In other words, is it possible that, although the eliciting emotional stimuli remain non-conscious, the (unaware) observer consciously feels the psychophysiological responses induced by these stimuli? There is initial evidence that this might be the case in patients with cortical blindness ${ }^{151}$. These patients, despite being unaware of the eliciting stimulus, reported negative feelings that were associated with activity in the somatosensory cortex.

Another aspect concerns the relationship between non-conscious perception of emotions and decisionmaking. Is it possible that when subjects perceive emotional stimuli non-consciously they unwittingly 'sense' the somatic changes elicited by the stimuli and use them as a guide to orient their decisions and preferences? This notion is reminiscent of the facial feedback hypothesis ${ }^{152}$ or of more elaborate theories about the relationship between bodily changes and decision-making, such as the somatic-marker hypothesis ${ }^{153}$. These issues are clearly crucial to fully exploring the potential influence of nonconscious perception of emotional stimuli in daily life, and attention needs to be paid in the future to the affective resonance and influence exerted by 'unseen' emotional signals over our conscious experience and our intentional behaviour towards the consciously perceived world. 
1. de Gelder, B., de Haan, E. \& Heywood, C. Out of Mind. Varieties of Unconscious Processes (Oxford Univ. Press, 2001)

2. de Gelder, B., Vroomen, J., Pourtois, G. \& Weiskrantz, L. Non-conscious recognition of affect in the absence of striate cortex. Neuroreport 10, 3759-3763 (1999). This study provided the first demonstration that patients with cortical blindness can perceive emotions non-consciously and can correctly guess the emotion displayed in facial expressions presented in the blind portion of their visual field.

Esteves, F., Dimberg, U. \& Ohman, A. Automatically elicited fear: conditioned skin conductance responses to masked facial expressions. Cogn. Emot. 8, 99-108 (1994).

One of the earliest studies showing that conscious perception of emotional faces can be blocked in healthy observers by backward masking, whereas non-conscious perception is still indexed by physiological changes in skin conductance (indicative of autonomic arousal).

4. Kunst-Wilson, W. R. \& Zajonc, R. B. Affective discrimination of stimuli that cannot be recognized. Science 207, 557-558 (1980).

5. LeDoux, J. E. The Emotional Brain (Simon \& Shuster, New York, 1996)

A very influential book summarizing evidence from the author's studies in animals indicating the existence of a direct subcortical pathway to the amygdala bypassing the primary sensory cortex in the rat brain. This book also fostered an evolution-inspired approach to non-conscious perception of emotions in neuroscience research.

6. Morris, J. S., Ohman, A. \& Dolan, R. J. Conscious and unconscious emotional learning in the human amygdala. Nature 393, 467-470 (1998).

7. Whalen, P. J. et al. Masked presentations of emotiona facial expressions modulate amygdala activity withou explicit knowledge. J. Neurosci. 18, 411-418 (1998). The first study reporting that fearful facia expressions that are masked still induce amygdala activity while activity in emotion-related cortical areas is suppressed.

8. Panksepp, J. Affective Neuroscience (Oxford Univ. Press, New York, 1998)

9. Williams, L. M. An integrative neuroscience model of 'significance' processing. J. Integr. Neurosci. 5, 1-47 (2006).

10. Posner, M. I. Attention: the mechanisms of consciousness. Proc. Natl Acad. Sci. USA 91 7398-7403 (1994)

11. Kentridge, R. W., Heywood, C. A. \& Weiskrantz, L. Spatial attention speeds discrimination without awareness in blindsight. Neuropsychologia $\mathbf{4 2}$, 831-835 (2004)

12. Dehaene, S., Changeux, J. P., Naccache, L., Sackur, J. $\&$ Sergent, C. Conscious, preconscious, and subliminal processing: a testable taxonomy. Trends Cogn. Sci. 10 204-211 (2006)

13. Savazzi, S. \& Marzi, C. A. Speeding up reaction time with invisible stimuli. Curr. Biol. 12, 403-407 (2002)

14. Koch, C. \& Tsuchiya, N. Attention and consciousness: two distinct brain processes. Trends Cogn. Sci. 11, 16-22 (2007).

15. Mack, A. \& Rock, I. Inattentional Blindness (MIT Press, Cambridge, Massachusetts, 1998).

16. Beck, D. M., Rees, G., Frith, C. D. \& Lavie, N. Neural correlates of change detection and change blindness. Nature Neurosci. 4, 645-650 (2001).

17. Vuilleumier, P. How brains beware: neural mechanisms of emotional attention. Trends Cogn. Sci. 9, 585-594 (2005).

18. Eastwood, J. D., Smilek, D. \& Merikle, P. M. Negative facial expression captures attention and disrupts performance. Percept. Psychophys. 65, 352-358 (2003).

19. Hart, S. J., Green, S. R., Casp, M. \& Belger, A. Emotional priming effects during Stroop task performance. Neuroimage 49, 2662-2670 (2010).

20. Georgiou, G. A. et al. Focusing on fear: attentional disengagement from emotional faces. Vis. Cogn. 12, 145-158 (2005)

21. Hansen, C. H. \& Hansen, R. D. Finding the face in the crowd: an anger superiority effect. J. Pers. Soc. Psychol. 54, 917-924 (1988).

22. Ohman, A., Flykt, A. \& Esteves, F. Emotion drives attention: detecting the snake in the grass. J. Exp. Psychol. Gen. 130, 466-478 (2001).

23. Anderson, A. K. Affective influences on the attentiona dynamics supporting awareness. J. Exp. Psychol. Gen. 134, 258-281 (2005).
24. Anderson, A. K. \& Phelps, E. A. Lesions of the human amygdala impair enhanced perception of emotionally salient events. Nature 411, 305-309 (2001). This study shows that enhanced processing of emotional signals under conditions of limited attention due to attentional blink is abolished in patients with amygdala lesions.

25. Anderson, A. K., Christoff, K., Panitz, D., De Rosa, E. $\&$ Gabrieli, J. D. Neural correlates of the automatic processing of threat facial signals. J. Neurosci. 23, 5627-5633 (2003)

26. Bishop, S. J., Duncan, J. \& Lawrence, A. D. State anxiety modulation of the amygdala response to unattended threat-related stimuli. J. Neurosci. 24 10364-10368 (2004).

27. Vuilleumier, P., Armony, J. L., Driver, J. \& Dolan, R. J. Effects of attention and emotion on face processing in the human brain: an event-related fMRI study. Neuron 30, 829-841 (2001).

This study used a dual-task design to induce attentional unawareness of facial expressions. The amygdala responded to fearful expressions regardless of the allocation of spatial attention whereas the fusiform face areas showed additive effects of attention and emotion, being more active when faces occurred at attended locations but always more activated by fearful than neutral faces even when faces were task-irrelevant and produced reduced cortical activity overall (see also reference 30 for contrasting results)

28. Williams, M. A., McGlone, F., Abbott, D. F. \& Mattingley, J. B. Differential amygdala responses to happy and fearful facial expressions depend on selective attention. Neuroimage 24, 417-425 (2005).

29. Pessoa, L. To what extent are emotional visual stimuli processed without attention and awareness? Curr Opin. Neurobiol. 15, 188-196 (2005).

30. Pessoa, L., McKenna, M., Gutierrez, E. \& Ungerleider, L. G. Neural processing of emotional faces requires attention. Proc. Natl Acad. Sci. USA 99 11458-11463 (2002)

31. Pessoa, L., Padmala, S. \& Morland, T. Fate of unattended fearful faces in the amygdala is determined by both attentional resources and cognitive modulation. Neuroimage 28, 249-255 (2005).

32. Silvert, L. et al. Influence of attentional demands on the processing of emotional facial expressions in the amygdala. Neuroimage 38, 357-366 (2007).

33. Vuilleumier, P. et al. Neural response to emotional faces with and without awareness: event-related fMRI in a parietal patient with visual extinction and spatial neglect. Neuropsychologia 40, 2156-2166 (2002)

34. Tamietto, M., Geminiani, G., Genero, R. \& de Gelder,

B. Seeing fearful body language overcomes attentional deficits in patients with neglect. J. Cogn. Neurosci. 19 , 445-454 (2007)

This study showed that fearful body expressions can be processed pre-attentively in patients with hemispatial neglect, thereby reducing the attentional bias and increasing perceptual sensitivity to stimuli presented in the left side of the space. Similar effects were previously reported for schematic facial expressions and spiders (see also references 36 and 37).

35. Tamietto, M. et al. Effects of emotional face cueing on line bisection in neglect: a single case study. Neurocase 11, 399-404 (2005).

36. Vuilleumier, P. \& Schwartz, S. Emotional facial expressions capture attention. Neurology 56 , 153-158 (2001).

37. Vuilleumier, P. \& Schwartz, S. Beware and be aware: capture of spatial attention by fear-related stimuli in neglect. Neuroreport 12,1119-1122 (2001).

38. Williams, M. A. \& Mattingley, J. B. Unconscious perception of non-threatening facial emotion in parietal extinction. Exp. Brain Res. 154, 403-406 (2004)

39. Macknik, S. L. \& Livingstone, M. S. Neuronal correlates of visibility and invisibility in the primate visual system. Nature Neurosci. 1, 144-149 (1998)

40. Tong, F., Meng, M. \& Blake, R. Neural bases of binocular rivalry. Trends Cogn. Sci. 10, 502-51 (2006)

41. Glascher, J. \& Adolphs, R. Processing of the arousal of subliminal and supraliminal emotional stimuli by the human amygdala. J. Neurosci. 23, 10274-10282 (2003)

42. Williams, L. M. et al. Mapping the time course of nonconscious and conscious perception of fear: an integration of central and peripheral measures. Hum Brain Mapp. 21, 64-74 (2004).
43. Liddell, B. J., Williams, L. M., Rathjen, J., Shevrin, H. \& Gordon, E. A temporal dissociation of subliminal versus supraliminal fear perception: an event-related potential study. J. Cogn. Neurosci. 16, 479-486 (2004).

44. Dimberg, U., Thunberg, M. \& Elmehed, K Unconscious facial reactions to emotional facial expressions. Psychol. Sci. 11, 86-89 (2000).

45. Tamietto, M. \& de Gelder, B. Emotional contagion for unseen bodily expressions: evidence from facial EMG. Automatic Face and Gesture Recognition (8th IEEE Int. Conference, Amsterdam, 2008)

46. Carlson, J. M., Reinke, K. S. \& Habib, R. A left amygdala mediated network for rapid orienting to masked fearful faces. Neuropsychologia 47, 1386-1389 (2009)

47. Critchley, H. D., Mathias, C. J. \& Dolan, R. J. Fear conditioning in humans: the influence of awareness and autonomic arousal on functional neuroanatomy. Neuron 33, 653-663 (2002).

48. Juruena, M. F. et al. Amygdala activation to masked happy facial expressions. J. Int. Neuropsychol. Soc. 16, 383-387 (2010)

49. Killgore, W. D. \& Yurgelun-Todd, D. A. Activation of the amygdala and anterior cingulate during nonconscious processing of sad versus happy faces. Neuroimage $\mathbf{2 1}$ 1215-1223 (2004)

50. Liddell, B. J. et al. A direct brainstem-amygdalacortical 'alarm' system for subliminal signals of fear. Neuroimage 24, 235-243 (2005).

51. Morris, J. S., Ohman, A. \& Dolan, R. J. A subcortical pathway to the right amygdala mediating 'unseen' fear. Proc. Natl Acad. Sci. USA 96, 1680-1685 (1999).

This study showed for the first time that non-conscious perception of emotions due to backward masking in healthy observers engages a subcortical pathway involving the superior colliculus, the pulvinar and the amygdala.

52. Whalen, P. J. et al. Human amygdala responsivity to masked fearful eye whites. Science 306, 2061 (2004).

53. Williams, L. M. et al. Mode of functional connectivity in amygdala pathways dissociates level of awareness for signals of fear. J. Neurosci. 26, 9264-9271 (2006).

This study showed that the conscious versus non-conscious perception of fearful facial expressions is associated with distinct patterns of functional connectivity. Conscious perception of fearful facial expressions was associated with negative functional connectivity between the amygdala and related cortical and subcortical areas, whereas non-conscious perception was supported by positive functional connectivity between the superior colliculus, the pulvinar and amygdala.

54. Williams, L. M. et al. Amygdala-prefrontal dissociation of subliminal and supraliminal fear. Hum. Brain Mapp. 27, 652-661 (2006)

55. Pasley, B. N., Mayes, L. C. \& Schultz, R. T. Subcortical discrimination of unperceived objects during binocular rivalry. Neuron 42, 163-172 (2004).

56. Williams, M. A., Morris, A. P., McGlone, F., Abbott, D. F. \& Mattingley, J. B. Amygdala responses to fearful and happy facial expressions under conditions of binocular suppression. J. Neurosci. 24, 2898-2904 (2004).

57. Yoon, K. L., Hong, S. W., Joormann, J. \& Kang, P. Perception of facial expressions of emotion during binocular rivalry. Emotion 9, 172-182 (2009).

58. Pessoa, L., Japee, S., Sturman, D. \& Ungerleider, L. G. Target visibility and visual awareness modulate amygdala responses to fearful faces. Cereb. Cortex 16 366-375 (2006)

59. Pessoa, L., Japee, S. \& Ungerleider, L. G. Visual awareness and the detection of fearful faces. Emotion 5, 243-247 (2005).

60. Tamietto, M. \& de Gelder, B. Affective blindsight in the intact brain: neural interhemispheric summation for unseen fearful expressions. Neuropsychologia 46 , 820-828 (2008)

61. Kim, M. J. et al. Behind the mask: the influence of mask-type on amygdala response to fearful faces. Soc Cogn. Affect. Neurosci. 10 Feb 2010 (doi:10.1093/ scan/nsq014).

62. de Gelder, B. et al. Intact navigation skills after bilateral loss of striate cortex. Curr. Biol. 18 , R1128-R1129 (2008).

63. de Gelder, B. Uncanny sight in the blind. Sci. Am. 302 60-65 (2010).

64. de Gelder, B. \& Hadjikhani, N. Non-conscious recognition of emotional body language. Neuroreport 17, 583-586 (2006). 
65. Hamm, A. O. et al. Affective blindsight: intact fear conditioning to a visual cue in a cortically blind patient. Brain 126, 267-275 (2003)

66. Morris, J. S., de Gelder, B., Weiskrantz, L. \& Dolan, R. J. Differential extrageniculostriate and amygdala responses to presentation of emotional faces in a cortically blind field. Brain 124, 1241-1252 (2001).

67. Pegna, A. J., Khateb, A., Lazeyras, F. \& Seghier, M. L. Discriminating emotional faces without primary visual cortices involves the right amygdala. Nature Neurosci. 8, 24-25 (2005)

68. Tamietto, M. et al. Unseen facial and bodily expressions trigger fast emotional reactions. Proc. Natl Acad. Sci. USA 106, 17661-17666 (2009). This study showed that spontaneous facial and pupillary reactions can be triggered by passive exposure to consciously and non-consciously perceived expressions of fear and happiness in patients with cortical blindness. Facial reactions were shown to be faster and pupil dilation higher for non-consciously than for consciously perceived facial and bodily expressions, whereas there was no difference between face and body stimuli.

69. de Gelder, B., Pourtois, G., van Raamsdonk, M., Vroomen, J. \& Weiskrantz, L. Unseen stimuli modulate conscious visual experience: evidence from interhemispheric summation. Neuroreport 12, 385-391 (2001).

70. Ro, T., Shelton, D., Lee, O. L. \& Chang, E. Extrageniculate mediation of unconscious vision in transcranial magnetic stimulation-induced blindsight. Proc. Natl Acad. Sci. USA 101, 9933-9935 (2004).

71. Jolij, J. \& Lamme, V. A. Repression of unconscious information by conscious processing: evidence from affective blindsight induced by transcranial magnetic stimulation. Proc. Natl Acad. Sci. USA 102. 10747-10751 (2005).

72. Hendler, T. et al. Sensing the invisible: differential sensitivity of visual cortex and amygdala to traumatic context. Neuroimage 19, 587-600 (2003).

73. Schiller, P. H. \& Malpeli, J. G. Properties and tectal projections of monkey retinal ganglion cells. J. Neurophysiol. 40, 428-445 (1977).

74. Tamietto, M. et al. Collicular vision guides nonconscious behavior. J. Cogn. Neurosci. 22, 888-902 (2010)

75. de Gelder, B., Morris, J. S. \& Dolan, R. J. Unconscious fear influences emotional awareness of faces and voices. Proc. Natl Acad. Sci. USA 102, 18682-18687 (2005).

76. Lyon, D. C., Nassi, J. J. \& Callaway, E. M. A disynaptic relay from superior colliculus to dorsal stream visual cortex in macaque monkey. Neuron 65, 270-279 (2010).

77. Ward, R., Danziger, S. \& Bamford, S. Response to visual threat following damage to the pulvinar. Curr. Biol. 15, 571-573 (2005).

78. Romanski, L. M., Giguere, M., Bates, J. F. \& Goldman-Rakic, P. S. Topographic organization of medial pulvinar connections with the prefrontal cortex in the rhesus monkey. J. Comp. Neurol. 379 , 313-332 (1997)

79. Phelps, E. A. \& LeDoux, J. E. Contributions of the amygdala to emotion processing: from animal models to human behavior. Neuron 48, 175-187 (2005).

80. Whalen, P. J. \& Phelps, E. A. (eds) The Human Amygdala (Guilford Press, New York, 2009).

81. Sergerie, K., Chochol, C. \& Armony, J. L. The role of the amygdala in emotional processing: a quantitative meta-analysis of functional neuroimaging studies. Neurosci. Biobehav. Rev. 32, 811-830 (2008).

82. Somerville, L. H., Kim, H., Johnstone, T., Alexander A. L. $\&$ Whalen, P. J. Human amygdala responses during presentation of happy and neutral faces: correlations with state anxiety. Biol. Psychiatry 55 897-903 (2004).

83. Jolkkonen, E., Pikkarainen, M., Kemppainen, S. \& Pitkanen, A. Interconnectivity between the amygdaloid complex and the amygdalostriatal transition area: a PHA-L study in rat. J. Comp. Neurol. 431, 39-58 (2001).

84. Whalen, P. J. et al. A functional MRI study of human amygdala responses to facial expressions of fear versus anger. Emotion 1, 70-83 (2001).

85. Whalen, P. J., Kapp, B. S. \& Pascoe, J. P. Neuronal activity within the nucleus basalis and conditioned neocortical electroencephalographic activation J. Neurosci. 14, 1623-1633 (1994)

86. Breiter, H. C. et al. Response and habituation of the human amygdala during visual processing of facial expression. Neuron 17, 875-887 (1996).
87. Berns, G. S., Cohen, J. D. \& Mintun, M. A. Brain regions responsive to novelty in the absence of awareness. Science 276, 1272-1275 (1997)

88. McHaffie, J. G., Stanford, T. R., Stein, B. E., Coizet, V. $\&$ Redgrave, P. Subcortical loops through the basal ganglia. Trends Neurosci. 28, 401-407 (2005)

89. Cauda, F. et al. in XIX Symposium Neuroradiologicum. Neuroradiol. J. (Centauro, Italy, 2010).

90. Mobbs, D. et al. When fear is near: threat imminence elicits prefrontal-periaqueductal gray shifts in humans. Science 317 , 1079-1083 (2007).

91. Aston-Jones, G., Chiang, C. \& Alexinsky, T. Discharge of noradrenergic locus coeruleus neurons in behaving rats and monkeys suggests a role in vigilance. Prog. Brain Res. 88, 501-520 (1991)

92. Bentley, P., Vuilleumier, P., Thiel, C. M., Driver, J. \& Dolan, R. J. Effects of attention and emotion on repetition priming and their modulation by cholinergic enhancement. J. Neurophysiol. 90, 1171-1181 (2003).

93. Adolphs, R. Neural systems for recognizing emotion. Curr. Opin. Neurobiol. 12, 169-177 (2002).

94. Calder, A. J., Lawrence, A. D. \& Young, A. W. Neuropsychology of fear and loathing. Nature Rev. Neurosci. 2, 352-363 (2001)

95. Phillips, M. L et al. Differential neural responses to overt and covert presentations of facial expressions of fear and disgust. Neuroimage 21, 1484-1496 (2004).

96. Nakamura, K. \& Ono, T. Lateral hypothalamus neuron involvement in integration of natural and artificial rewards and cue signals. J. Neurophysiol. 55 163-181 (1986)

97. Tamietto, M., Pullens, P., Weiskrantz, L., Goebel, R. \& de Gelder, B. in Second Meeting of the Federation of the European Societies of Neuropsychology (ESN, The Netherlands, 2010).

98. Krolak-Salmon, P., Henaff, M. A., Vighetto, A. Bertrand, O. \& Mauguiere, F. Early amygdala reaction to fear spreading in occipital, temporal, and frontal cortex: a depth electrode ERP study in human. Neuron 42, 665-676 (2004).

99. Leppanen, J. M., Moulson, M. C., Vogel-Farley, V. K. \& Nelson, C. A. An ERP study of emotional face processing in the adult and infant brain. Child Dev. 78 232-245 (2007).

100. Holmes, A. Vuilleumier, P. \& Eimer, M. The processing of emotional facial expression is gated by spatial attention: evidence from event-related brain potentials. Brain Res. Cogn. Brain Res. 16, 174-184 (2003)

101. Luo, Q Holroyd, T. Jones, M., Hendler, T \& Blair, J. Neural dynamics for facial threat processing as revealed by $\gamma$ band synchronization using MEG. Neuroimage 34, 839-847 (2007)

102. Livingstone, M. $\&$ Hubel, D. Segregation of form, color, movement, and depth: anatomy, physiology, and perception. Science 240, 740-749 (1988).

103. Vuilleumier, P., Armony, J. L., Driver, J. \& Dolan, R. J. Distinct spatial frequency sensitivities for processing faces and emotional expressions. Nature Neurosci. 6 , 624-631 (2003).

104. Jarvis, E. D. et al. Avian brains and a new understanding of vertebrate brain evolution. Nature Rev. Neurosci. 6, 151-159 (2005).

105. Andrew, R. J. Changes in visual responsiveness following intercollicular lesions and their effects on avoidance and attack. Brain Behav. Evol. 10, 400-424 (1975)

106. Linke, R., De Lima, A. D., Schwegler, H. \& Pape, H. C. Direct synaptic connections of axons from superior colliculus with identified thalamo-amygdaloid projection neurons in the rat: possible substrates of a subcortical visual pathway to the amygdala. J. Comp. Neurol. 403, 158-170 (1999).

107. Cohen, J. D. \& Castro-Alamancos, M. A. Early sensory pathways for detection of fearful conditioned stimuli: tectal and thalamic relays. J. Neurosci. 27 7762-7776 (2007)

108. Weiskrantz, L. Behavioral changes associated with ablation of the amygdaloid complex in monkeys. J. Comp. Physiol Psychol 49, 381-391 (1956).

109. Jones, E. G. \& Burton, H. A projection from the medial pulvinar to the amygdala in primates. Brain Res. 104, 142-147 (1976)

110. Harting, J. K., Huerta, M. F., Hashikawa, T \& van Lieshout, D. P. Projection of the mammalian superior colliculus upon the dorsal lateral geniculate nucleus: organization of tectogeniculate pathways in ninetee species. J. Comp. Neurol. 304, 275-306 (1991).

111. Schmid, M. C. et al. Blindsight depends on the lateral geniculate nucleus. Nature 466, 373-377 (2010)
112. Isbell, L. A. Snakes as agents of evolutionary change in primate brains. J. Hum. Evol. 51, 1-35 (2006)

113. Leppanen, J. M. \& Nelson, C. A. Tuning the developing brain to social signals of emotions. Nature Rev. Neurosci. 10, 37-47 (2009).

114. de Gelder, B. et al. Beyond the face: exploring rapid influences of context on face processing. Prog. Brain Res. 155, 37-48 (2006).

115. Johnson, M. H. Subcortical face processing. Nature Rev. Neurosci. 6, 766-774 (2005).

116. Rossion, B., de Gelder, B., Pourtois, G., Guerit, J. M. \& Weiskrantz, L. Early extrastriate activity without primary visual cortex in humans. Neurosci. Lett. 279 25-28 (2000)

117. Tamietto, M., Adenzato, M., Geminiani, G. \& de Gelder, B. Fast recognition of social emotions takes the whole brain: interhemispheric cooperation in the absence of cerebral asymmetry. Neuropsychologia 45, 836-843 (2007)

118. de Gelder, B. Towards the neurobiology of emotiona body language. Nature Rev. Neurosci. 7, 242-249 (2006).

119. de Gelder, B. et al. Standing up for the body. Recent progress in uncovering the networks involved in the perception of bodies and bodily expressions. Neurosci. Biobehav. Rev. 34, 513-527 (2010).

120. Alpers, G. W. et al. Attention and amygdala activity: an fMRI study with spider pictures in spider phobia. J. Neural Transm. 116, 747-757 (2009).

121. Carlsson, K et al. Fear and the amygdala: manipulation of awareness generates differential cerebral responses to phobic and fear-relevant (but nonfeared) stimuli. Emotion 4, 340-353 (2004).

122. Wendt, J., Lotze, M., Weike, A. I., Hosten, N. \& Hamm, A. O. Brain activation and defensive response mobilization during sustained exposure to phobiarelated and other affective pictures in spider phobia. Psychophysiology 45, 205-215 (2008)

123. de Gelder, B., Pourtois, G. \& Weiskrantz, Fear recognition in the voice is modulated by unconsciously recognized facial expressions but not by unconsciously recognized affective pictures. Proc. Natl Acad. Sci. USA 99, 4121-4126 (2002).

124. van Honk, J. et al. Baseline salivary cortisol levels and preconscious selective attention for threat. A pilot study. Psychoneuroendocrinology 23, 741-747 (1998).

125. Ruiz-Padial, E., Mata, J. L., Rodriguez, S., Fernandez, M. C. \& Vila, J. Non-conscious modulation of cardiac defense by masked phobic pictures. Int J. Psychophysiol. 56, 271-281 (2005).

126. Frith, C. Role of facial expressions in social interactions. Phil. Trans. R. Soc. Lond. B 364 , 3453-3458 (2009).

127. Rinn, W. E. The neuropsychology of facial expression: a review of the neurological and psychological mechanisms for producing facial expressions Psychol. Bull. 95, 52-77 (1984).

128. Ladavas, E., Cimatti, D., Del Pesce, M. \& Tuozzi, G. Emotional evaluation with and without conscious stimulus identification: evidence from a split-brain patient. Cogn. Emot. 7, 95-114 (1993).

129. Rees, G., Kreiman, G. \& Koch, C. Neural correlates of consciousness in humans. Nature Rev. Neurosci. 3 261-270 (2002)

130. Dehaene, S. et al. Cerebral mechanisms of word masking and unconscious repetition priming. Nature Neurosci. 4, 752-758 (2001)

131. Moutoussis, K. \& Zeki, S. The relationship between cortical activation and perception investigated with invisible stimuli. Proc. Natl Acad. Sci. USA 99 9527-9532 (2002).

132. Moutoussis, K. $\&$ Zeki, S. Seeing invisible motion: a human FMRI study. Curr. Biol. 16, 574-579 (2006).

133. Azzopardi, P. \& Cowey, A. Is blindsight like normal, near-threshold vision? Proc. Natl Acad. Sci. USA 94 14190-14194 (1997).

134. Meeres, S. L. \& Graves, R. E. Localization of unseen visual stimuli by humans with normal vision. Neuropsychologia 28, 1231-1237 (1990).

135. Leopold, D. A. \& Logothetis, N. K. Activity changes in early visual cortex reflect monkeys' percepts during binocular rivalry. Nature 379, 549-553 (1996).

136. Sergent, C., Baillet, S. \& Dehaene, S. Timing of the brain events underlying access to consciousness during the attentional blink. Nature Neurosci. 8 1391-1400 (2005).

137. Haynes, J. D. \& Rees, G. Predicting the orientation of invisible stimuli from activity in human primary visual cortex. Nature Neurosci. 8, 686-691 (2005). 
138. Rees, G. et al. Unconscious activation of visual cortex in the damaged right hemisphere of a parietal patient with extinction. Brain 123, 1624-1633 (2000).

139. Sahraie, A. et al. Pattern of neuronal activity associated with conscious and unconscious processing of visual signals. Proc. Natl Acad. Sci. USA 94, 9406-9411 (1997).

140. Bush, P. \& Sejnowski, T. Inhibition synchronizes sparsely connected cortical neurons within and between columns in realistic network models. J. Comput. Neurosci. 3, 91-110 (1996).

141. Lamme, V. A. \& Roelfsema, P. R. The distinct modes of vision offered by feedforward and recurrent processing. Trends Neurosci. 23, 571-579 (2000).

142. Anders, S. et al. When seeing outweighs feeling: a role for prefrontal cortex in passive control of negative affect in blindsight. Brain 132, 3021-3031 (2009)

143. Niedenthal, P. M. Implicit perception of affective information. J. Exp. Soc. Psychol. 26, 505-527 (1990).

144. Tamietto, M., Latini Corazzini, L., de Gelder, B. \& Geminiani, G. Functional asymmetry and interhemispheric cooperation in the perception of emotions from facial expressions. Exp. Brain Res. 171, 389-404 (2006).

145. Amaral, D. G., Behniea, H. \& Kelly, J. L. Topographic organization of projections from the amygdala to the visual cortex in the macaque monkey. Neuroscience 118, 1099-1120 (2003)

146. Rodman, H. R., Gross, C. G. \& Albright, T. D. Afferent basis of visual response properties in area Montana of the macaque. II. Effects of superior colliculus removal. J. Neurosci. 10, 1154-1164 (1990).

147. Morris, J. S. et al. A neuromodulatory role for the human amygdala in processing emotional facial expressions. Brain 121, 47-57 (1998). This positron emission tomography study showed that activation in extrastriate visual areas in response to task-irrelevant fearful faces is modulated by amygdala activity, thereby providing the first evidence that subcortical structures involved in processing emotional stimuli can modulate directly cortical activity.
148. Peelen, M. V., Atkinson, A. P. Andersson, F \& Vuilleumier, P. Emotional modulation of body-selective visual areas. Soc. Cogn. Affect. Neurosci. 2, 274-283 (2007).

149. Vuilleumier, P., Richardson, M. P., Armony, J. L., Driver, J. \& Dolan, R. J. Distant influences of amygdala lesion on visual cortical activation during emotional face processing. Nature Neurosci. 7, 1271-1278 (2004).

150. Craig, A. D. How do you feel--now? The anterior insula and human awareness. Nature Rev. Neurosci. 10 59-70 (2009).

151. Anders, S. et al. Parietal somatosensory association cortex mediates affective blindsight. Nature Neurosci. 7, 339-340 (2004).

This study shows that patients with cortical blindness can report negative feelings induced by the presentation of aversively-conditioned facial expressions in their blind visual field, despite being unaware of the origin of these feelings. This effect was associated with enhanced activity in the parietal somatosensory cortex.

152. Tomkins, S. S. Affect, Imagery, Consciousness (Springer, New York, 1962-1963).

153. Damasio, A. R. Descartes' Error: Emotion, Reason, and the Human Brain (G. P. Putnam's Sons, New York, 1994)

154. Merikle, P. M., Smilek, D. \& Eastwood, J. D. Perception without awareness: perspectives from cognitive psychology. Cognition 79, 115-134 (2001).

155. Wiens, S. Subliminal emotion perception in brain imaging: findings, issues, and recommendations. Prog. Brain Res. 156, 105-121 (2006)

156. Cheesman, J. \& Merikle, P. M. Distinguishing conscious from unconscious perceptual processes. Can. J. Psychol. 40, 343-367 (1986).

157. Simion, F., Valenza, E., Umilta, C. \& Dalla Barba, B. Preferential orienting to faces in newborns: a temporal-nasal asymmetry. J. Exp. Psychol. Hum. Percept. Perform. 24, 1399-1405 (1998)

158. Kotsoni, E., de Haan, M. \& Johnson, M. H. Categorical perception of facial expressions by 7-month-old infants. Perception 30, 1115-1125 (2001)
159. Peltola, M. J., Leppanen, J. M., Palokangas, T. \& Hietanen, J. K. Fearful faces modulate looking duration and attention disengagement in 7-month-old infants. Dev. Sci. 11, 60-68 (2008).

160. Kordower, J. H., Piecinski, P. \& Rakic, P. Neurogenesis of the amygdaloid nuclear complex in the rhesus monkey. Brain Res. Dev. Brain Res. 68, 9-15 (1992).

161. Tinti, C., Adenzato, M., Tamietto, M. \& Cornoldi, C. Visual experience is not necessary for efficient survey spatial cognition: evidence from blindness. Q. J. Exp. Psychol. (Colchester) 59, 1306-1328 (2006).

162. Baron-Cohen, S. et al. The amygdala theory of autism Neurosci. Biobehav Rev. 24, 355-364 (2000)

163. Deruelle, C., Rondan, C., Gepner, B. \& Tardif, C. Spatial frequency and face processing in children with autism and Asperger syndrome. J. Autism Dev. Disord. 34, 199-210 (2004)

Acknowledgements

We gratefully acknowledge valuable comments from P. Whalen and two anonymous referees. M.T. was supported by a Veni grant (451-07-032) from the Netherlands Organization for Scientific Research (NWO) and also partly supported by the Fondazione Carlo Molo, Turin, Italy and by a Bando Scienze Umane e Sociali 2008 grant from the Regione Piemonte, Italy, for the project Grammatica Invisibile delle Relazioni Sociali (GIRS) (grant number 4). B.d.G. was supported by an Future and Emerging Technologies (FET) Open Scheme grant (ICT-2009-C) from the European Union for the Emotional Interaction Grounded in Realistic Context (also known as TANGO) Project (249858) and by a Lagrange Fellow visiting professorship to the University of Torino, Turin, Italy funded by the Cassa di Risparmio di Torino (CRT) Foundation.

Competing interests statement

The authors declare no competing financial interests.

FURTHER INFORMATION

Beatrice de Gelder's homepage:

http://www.beatricedegelder.com

ALL LINKS ARE ACTIVE IN THE ONLINE PDF 